\title{
MULTIPLE ZEROS OF DEDEKIND ZETA FUNCTIONS
}

\author{
JERZY BROWKIN
}

To the memory of Emil Artin on the 50-th anniversary of his death

\begin{abstract}
It is proved that there are Dedekind's zeta functions with multiple zeros in the critical strip of arbitrarily large multiplicity. There are given examples of such zeros of the Dedekind zeta function of the field $\mathbb{Q}\left(\zeta_{3}, \sqrt[3]{5}\right)$.
\end{abstract}

Keywords: Dedekind's zeta functions, Artin's $L$-functions, zeros in the critical strip, induced characters.

\section{Introduction}

It is conjectured that all zeros in the critical strip $0<\operatorname{Re} s<1$ of Riemann's zeta function lie on the critical line $\operatorname{Re} s=\frac{1}{2}$ and are simple. The conjecture is supported by strong numerical evidence.

On the other hand, there are known fields $F$ such that the Dedekind zeta function $\zeta_{F}(s)$ has a zero at $s=\frac{1}{2}$ (see $[\mathrm{A}],[\mathrm{F}]$ ). From the functional equation it follows that such a zero has an even multiplicity, so it cannot be simple.

In the present paper we prove that for some finite groups $G$ for every Galois extension $F / E$ of number fields with the Galois group $G$ the Dedekind zeta function $\zeta_{F}(s)$ has infinitely many multiple zeros in the critical strip $0<\operatorname{Re} s<1$. Moreover, for an appropriate group $G$ such a zero can have an arbitrarily large multiplicity. In the case of $G=S_{3}$ and $E=\mathbb{Q}$, applying results of [W], we prove that there are infinitely many multiple zeros of $\zeta_{F}(s)$ lying on the critical line.

We give some numerical examples.

Finally, we show, applying the Brauer-Kuroda relations, how to obtain the Dedekind zeta functions with multiple zeros in the critical strip in the case of some other groups $G$.

2010 Mathematics Subject Classification: primary: 11M26; secondary: 20C15 


\section{Groups}

Let $G$ be the following group of matrices over $\mathbb{F}_{q}$, where $q>2$ is a power of prime:

$$
G:=\left\{\left(\begin{array}{cc}
a & b \\
0 & 1
\end{array}\right): a \in \mathbb{F}_{q}^{*}, b \in \mathbb{F}_{q}\right\}
$$

The subgroups of $G$ :

$$
A:=\left\{\left(\begin{array}{ll}
1 & b \\
0 & 1
\end{array}\right): b \in \mathbb{F}_{q}\right\} \quad \text { and } \quad H:=\left\{\left(\begin{array}{cc}
a & 0 \\
0 & 1
\end{array}\right): a \in \mathbb{F}_{q}^{*}\right\}
$$

are isomorphic to the additive and multiplicative groups of the field $\mathbb{F}_{q}$, respectively.

There is the exact sequence

$$
1 \longrightarrow A \longrightarrow G \stackrel{\varphi}{\longrightarrow} \mathbb{F}_{q}^{*} \longrightarrow 1
$$

where $\varphi\left(\left(\begin{array}{ll}a & b \\ 0 & 1\end{array}\right)\right)=a$. Moreover, $\varphi: H \rightarrow \mathbb{F}_{q}^{*}$ is an isomorphism.

By the standard arguments (it is an easy exercise) one can prove:

Claim 2.1. The commutator subgroup $G^{\prime}$ of $G$ is $A$.

Claim 2.2. For $g:=\left(\begin{array}{ll}a & b \\ 0 & 1\end{array}\right)$ the coset $g A$ equals $\left\{\left(\begin{array}{ll}a & x \\ 0 & 1\end{array}\right): x \in \mathbb{F}_{q}^{*}\right\}$. So there are $q-1$ cosets determined by the values of $a \in \mathbb{F}_{q}^{*}$.

Claim 2.3. In $G$ there are $q$ conjugacy classes: $\{I\}$, where $I$ is the unit matrix, $\left\{\left(\begin{array}{ll}1 & b \\ 0 & 1\end{array}\right): b \in \mathbb{F}_{q}^{*}\right\}=A \backslash\{I\}$, and $q-2$ cosets $g A$, where $g \notin A$.

\section{Characters}

Let $\lambda_{1}$ be the principal character of the trivial subgroup $1=\{I\}$. Since the group $H$ is abelian of order $q-1$, there are $q-1$ irreducible characters $\varphi_{1}, \varphi_{2}, \ldots, \varphi_{q-1}$ of $H$, which are 1-dimensional. Let $\varphi_{1}$ be principal.

Similarly, let $\psi_{1}, \psi_{2}, \ldots, \psi_{q}$ be irreducible characters of the group $A$, where $\psi_{1}$ is principal. They are 1-dimensional.

By Claim 2.1, the commutator subgroup $G^{\prime}$ of $G$ has index $q-1$. Therefore there are $q-1$ 1-dimensional irreducible characters $\chi_{1}, \chi_{2}, \ldots, \chi_{q-1}$ of $G$, let $\chi_{1}$ be principal.

By Claim 2.3, there is one more irreducible character $\chi_{q}$ of $G$. Its dimension $\chi_{q}(1)$ can be determined from the equality

$$
\sum_{j=1}^{q} \chi_{j}(1)^{2}=\# G
$$

We get $\chi_{q}(1)^{2}=\# G-\sum_{j=1}^{q-1} \chi_{j}(1)^{2}=q(q-1)-(q-1)=(q-1)^{2}$. Hence $\chi_{q}(1)=q-1>1$, since $q>2$, by assumption. 
Lemma 3.1. The following formulas for induced characters hold:

(i) $\operatorname{ind}_{1}^{G}\left(\lambda_{1}\right)=\sum_{j=1}^{q} \chi_{j}(1) \chi_{j}=\sum_{j=1}^{q-1} \chi_{j}+(q-1) \chi_{q}$.

(ii) $\operatorname{ind}_{H}^{G}\left(\varphi_{1}\right)=\chi_{1}+\chi_{q}$.

(iii) $\operatorname{ind}_{A}^{G}\left(\psi_{j}\right)=\chi_{q}$ for every $j=2, \ldots, q$.

Proof. The formula (i) is well known, see [H, (3.9)] or [T, (1.5.7)].

(ii) Obviously, $\operatorname{ind}_{H}^{G}\left(\varphi_{1}\right)$ is a summand of $\operatorname{ind}_{1}^{G}\left(\lambda_{1}\right)$ and $\chi_{1}$ is a summand of $\operatorname{ind}_{H}^{G}\left(\varphi_{1}\right)$. From

$$
\left.\sum_{j=1}^{q-1} \chi_{j}(1)=q-1 \quad \text { and } \quad \operatorname{(ind}_{H}^{G}\left(\varphi_{1}\right)\right)(1)=(G: H) \varphi_{1}(1)=(G: H)=q
$$

we conclude that $\operatorname{ind}_{H}^{G}\left(\varphi_{1}\right)$ is not a summand of $\sum_{j-1}^{q-1} \chi_{j}$. Consequently, $\chi_{q}$ is a summand of $\operatorname{ind}_{H}^{G}\left(\varphi_{1}\right)$.

Comparing the dimensions

$$
\left.\operatorname{(ind}_{H}^{G}\left(\varphi_{1}\right)\right)(1)=q \quad \text { and } \quad\left(\chi_{1}+\chi_{q}\right)(1)=1+(q-1)=q
$$

we get (ii).

(iii) The proof is similar. Since $\chi_{1}$ is a summand of $\operatorname{ind}_{A}^{G}\left(\psi_{1}\right)$ and $\left(\operatorname{ind}_{A}^{G}\left(\psi_{1}\right)\right)(1)$ $=(G: A)=q-1$, we conclude that $\chi_{q}$ is not a summand of $\operatorname{ind}_{A}^{G}\left(\psi_{1}\right)$. Consequently, comparing dimensions we get

$$
\operatorname{ind}_{A}^{G}\left(\psi_{1}\right)=\sum_{j=1}^{q-1} \chi_{j}
$$

and hence $\operatorname{ind}_{A}^{G}\left(\psi_{j}\right)=\chi_{q}$ for every $j=1,2, \ldots, q-1$.

\section{Artin's $L$-functions}

Let the group $G$ defined above be the Galois group of some Galois extension $F / E$ of number fields. Let $K$ and $M$ be subfields corresponding to the subgroups $H$ and $A$, respectively, i.e. $K=F^{H}, M=F^{A}$.

Applying the well known properties of Artin's $L$-functions (see [H]) corresponding to characters defined above we get the following lemmas.

Lemma 4.1. In the above notation we have

$$
\begin{array}{ll}
L\left(s, \lambda_{1}, F / F\right)=\zeta_{F}(s), & L\left(s, \varphi_{1}, F / K\right)=\zeta_{K}(s), \\
L\left(s, \psi_{1}, F / M\right)=\zeta_{M}(s), & L\left(s, \chi_{1}, F / E\right)=\zeta_{E}(s) .
\end{array}
$$

Proof. The Artin $L$-function corresponding to a principal character is the Dedekind zeta function of an appropriate field. 
Lemma 4.2. The following Artin's L-functions are classical abelian L-series of corresponding fields:

$$
\begin{array}{cl}
L\left(s, \varphi_{j}, F / K\right), & 2 \leqslant j \leqslant q-1, \\
L\left(s, \psi_{j}, F / M\right), & 2 \leqslant j \leqslant q, \\
L\left(s, \chi_{j}, F / E\right), & 2 \leqslant j \leqslant q-1 .
\end{array}
$$

Proof. The Artin $L$-function corresponding to a non-principal 1-dimensional character is the classical abelian $L$-series of the appropriate field.

\section{Lemma 4.3.}

(i) $\zeta_{F}(s)=\zeta_{E}(s) \prod_{j=2}^{q-1} L\left(s, \chi_{j}, F / E\right) \cdot L\left(s, \chi_{q}, F / E\right)^{q-1}$.

(ii) $\zeta_{K}(s)=\zeta_{E}(s) \cdot L\left(s, \chi_{q}, F / E\right)$.

(iii) $L\left(s, \psi_{j}, F / M\right)=L\left(s, \chi_{q}, F / E\right)$ for $j=2, \ldots, q$.

Proof. In the proof we apply several times the multiplicativity of Artin's $L$-functions:

$$
L\left(s, \nu_{1}+\nu_{2}, R / S\right)=L\left(s, \nu_{1}, R / S\right) \cdot L\left(s, \nu_{2}, R / S\right)
$$

where $\nu_{1}, \nu_{2}$ are characters of the Galois group of the Galois extension $R / S$ of number fields.

Moreover, we apply the invariance of Artin's $L$-functions with respect to the inducing of characters:

$$
L\left(s, \operatorname{ind}_{U}^{V}(\nu), R / S\right)=L(s, \nu, R / T),
$$

where $R \supset T \supset S, R / S$ is a Galois extension, $U=\operatorname{Gal}(R / T), V=\operatorname{Gal}(R / S)$ and $\nu$ is a character of $U$.

The Lemma follows from Lemma 3.1 (i), (ii) and (iii), respectively, and Lemma 4.1.

\section{Multiple zeros of Dedekind zeta functions}

Theorem 5.1. The Dedekind zeta function $\zeta_{F}(s)$ of the field $F$ defined above has infinitely many zeros of multiplicities $\geqslant q-1$ in the critical strip.

Proof. It is known that a classical abelian $L$-series corresponding to a nonprincipal 1-dimensional character is an entire function (it does not have poles). It is also known that it has infinitely many zeros in the critical strip.

By Lemma 4.3(iii) and Lemma 4.2, $L\left(s, \chi_{q}, F / E\right)$ is a classical abelian $L$-series corresponding to a non-principal 1-dimensional character $\psi_{j}, j \neq 1$.

Similarly, by Lemma $4.2, L\left(s, \chi_{j}, F / E\right)$ for $j=2, \ldots, q-1$, are classical abelian $L$-series corresponding to the non-principal 1-dimensional characters $\chi_{j}$.

Therefore on the r.h.s. of the formula in Lemma 4.3(i) the only pole is the pole of $\zeta_{E}(s)$ at $s=1$.

It follows, by Lemma 4.3(i), that every zero of $L\left(s, \chi_{q}, F / E\right)$ is a zero of multiplicity at least $q-1$ of $\zeta_{F}(s)$. In particular, the same holds for zeros in the critical strip. 
Theorem 5.2. Let $F$ be a Galois extension of $\mathbb{Q}$ with the Galois group $S_{3}$. Then infinitely many multiple zeros of $\zeta_{F}(s)$ lie on the critical line.

Proof. In the above notation assume that $q=3$. Then $G=S_{3}$ and $K=F^{\langle(12)\rangle}$ is a cubic subfield of $F$, which is not Galois.

The irreducible character $\chi_{3}$ of $S_{3}$ is 2-dimensional, and from Lemma 4.3(i),(ii) we get

$$
\begin{aligned}
\zeta_{F}(s) & =\zeta(s) \cdot L\left(s, \chi_{2}, F / \mathbb{Q}\right) \cdot L\left(s, \chi_{3}, F / \mathbb{Q}\right)^{2}, \\
\zeta_{K}(s) & =\zeta(s) \cdot L\left(s, \chi_{3}, F / \mathbb{Q}\right) .
\end{aligned}
$$

In [W] it is proved that the number $N_{0}(T)$ of zeros $s=\frac{1}{2}+i t$ with $0 \leqslant t \leqslant T$ (counted with their multiplicities) of $\zeta_{K}(s) / \zeta(s)=L\left(s, \chi_{3}, F / \mathbb{Q}\right)$ satisfies

$$
N_{0}(T)>\frac{T^{1 / 2}}{(\log T)^{2 / 3}(\log \log T)^{(1 / 3)+\varepsilon}} \quad \text { for } \quad T>T_{0}(\varepsilon) .
$$

By (1), such zeros are multiple zeros of $\zeta_{F}(s)$, hence the theorem follows.

Other numerical examples which lead to multiple zeros in the critical strip of Dedekind zeta functions are given in [To]. There are considered fields of degrees up to 6 .

\section{The functional equation}

In general it is difficult to write explicitly the functional equation for an Artin $L$-function. In the case of $L\left(s, \chi_{q}, F / E\right)$ it is easy due to Lemma 4.3(ii). Namely, it is sufficient to divide side by side the functional equations for $\zeta_{K}(s)$ and for $\zeta_{E}(s)$.

For

$$
\xi_{K}(s):=\left(\frac{|d(K)|}{\pi^{(K: \mathbb{Q})}}\right)^{s / 2} \Gamma\left(\frac{s}{2}\right)^{r_{1}(K)+r_{2}(K)} \Gamma\left(\frac{s+1}{2}\right)^{r_{2}(K)} \zeta_{K}(s)
$$

and

$$
\xi_{E}(s):=\left(\frac{|d(E)|}{\pi^{(E: \mathbb{Q})}}\right)^{s / 2} \Gamma\left(\frac{s}{2}\right)^{r_{1}(E)+r_{2}(E)} \Gamma\left(\frac{s+1}{2}\right)^{r_{2}(E)} \zeta_{E}(s)
$$

we have the functional equations

$$
\xi_{K}(s)=\xi_{K}(1-s) \quad \text { and } \quad \xi_{E}(s)=\xi_{E}(1-s)
$$

Dividing these equations side by side we get, by Lemma 4.3(ii),

$$
\begin{aligned}
\xi_{K / E}(s):= & \frac{\xi_{K}(s)}{\xi_{E}(s)}=\left(\frac{|d(K)|}{|d(E)| \pi^{(K: E)}}\right)^{s / 2} \Gamma\left(\frac{s}{2}\right)^{r_{1}(K)+r_{2}(K)-r_{1}(E)-r_{2}(E)} \\
& \times \Gamma\left(\frac{s+1}{2}\right)^{r_{2}(K)-r_{2}(E)} L\left(s, \chi_{q}, F / E\right) .
\end{aligned}
$$


Therefore the functional equation for $L\left(s, \chi_{q}, F / E\right)$ is

$$
\xi_{K / E}(s)=\xi_{K / E}(1-s)
$$

It follows that the function $L\left(s, \chi_{q}, F / E\right)$ belongs to the Selberg class, and its degree is

$$
\begin{aligned}
\left(r_{1}(K)+r_{2}(K)-r_{1}(E)\right. & \left.-r_{2}(E)\right)+\left(r_{2}(K)-r_{2}(E)\right)=(K: \mathbb{Q})-(E: \mathbb{Q}) \\
= & (E: \mathbb{Q})((K: E)-1)=(q-1)(E: \mathbb{Q})=\chi_{q}(1)(E: \mathbb{Q}) .
\end{aligned}
$$

\section{Conjectures}

All observations made above concern fields with very special Galois groups $G$. One may expect that analogous results hold for Galois fields with an arbitrary nonabelian Galois group. We state corresponding conjectures in a more precise form.

Let $F / E$ be a Galois extension of number fields with the Galois group $G$, which is not abelian, and let $L(s, \chi, F / E)$ be the Artin L-function where $\chi$ is an irreducible character of $G$ of dimension $\chi(1)>1$.

Conjectures. $\quad$ 1. The Dedekind zeta function $\zeta_{F}(s)$ has infinitely many multiple zeros in the critical strip (and even on the critical line).

2. $L(s, \chi, F / E)$ belongs to the Selberg class, and is of degree $\chi(1) \cdot(E: \mathbb{Q})$.

\section{Numerical examples}

We choose the group $G$ and the field $F$ as simple as possible. Namely, let $q=3$. There is a unique non-abelian group of order $q(q-1)=6$, the symmetric group $G=S_{3}$.

Let $E=\mathbb{Q}$ and $F=\mathbb{Q}\left(\zeta_{3}, \sqrt[3]{d}\right)$, for some cubefree $d>1$. Then $\operatorname{Gal}(F / \mathbb{Q})=S_{3}$.

We are looking for zeros on the critical line of the $\operatorname{Artin} L$-function $L\left(s, \chi_{3}, F / \mathbb{Q}\right)$, where $\chi_{3}$ is the unique irreducible character of dimension 2 of the group $S_{3}$.

We have $K=\mathbb{Q}(\sqrt[3]{d})$. Therefore, by Lemma 4.3(ii), it is sufficient to look for zeros of $\zeta_{K}(s)$ which are not zeros of the Riemann zeta function $\zeta_{\mathbb{Q}}(s)=\zeta(s)$.

In the cases of $d=2,3,6$ and 12 some such zeros have been computed by Lagarias and Odlyzko in [LO, Table 1].

Using the package GP/PARI one can easily give (numerically) much more such zeros also for other values of $d$. E.g. we give zeros $s=\frac{1}{2}+i t$ of $\zeta_{K}(s)$ in the case $d=5$, i.e. $F=\mathbb{Q}\left(\zeta_{3}, \sqrt[3]{5}\right)$, which belong to the critical line and lie below the first zero of the Riemann zeta function $s=\frac{1}{2}+14.134725 i$. From the above it follows that they are zeros of $\zeta_{F}(s)$ of the multiplicities at least 2.

Multiple zeros $s=\frac{1}{2}+i t$ of $\zeta_{F}(s)$ are given by the following values of $t<$ 14.134725: 

1.690758517
3.117579654
5.605049571
6.378094679
6.924439738
8.156647047
8.913454288
10.021397746
10.804860136
11.340000786
12.400920955
13.020090963
13.786318772

\section{Brauer-Kuroda relations}

For a Galois extension $F / E$ of number fields with the non-exceptional Galois group $G$ there are known multiplicative relations between $\zeta_{F}(s)$ and the Dedekind zeta functions of some intermediate fields $F^{H}$, for cyclic subgroups $H$ of $G$, see [BBX].

If in such a relation some zeta functions appear with exponents $>1$, one may expect that $\zeta_{F}(s)$ has multiple zeros in the critical strip.

We illustrate this by the following example. For simplicity, we denote by $\zeta_{\tau}(s)$ the Dedekind zeta function of the subfield of $F$ fixed by the automorphism $\tau \in G$.

Example. For the following groups $G$ we have the corresponding Brauer-Kuroda relations:

(i) $G=S_{3}, \quad \zeta_{F}(s) \cdot \zeta_{E}(s)^{2}=\zeta_{(123)}(s) \zeta_{(12)}(s)^{2}$,

(ii) $G=A_{4}, \quad \zeta_{F}(s) \cdot \zeta_{E}(s)^{2}=\zeta_{(12)(34)}(s) \zeta_{(234)}(s)^{2}$,

(iii) $G=S_{5}, \quad \zeta_{F}(s) \cdot \zeta_{E}(s)^{4}=\zeta_{(123)(45)}(s)^{2} \cdot \zeta_{(1234)}(s)^{2} \cdot \zeta_{(12345)}(s)$,

(iv) $G=D_{2 p}, \zeta_{F}(s) \cdot \zeta_{E}(s)^{2}=\zeta_{\sigma_{p}}(s) \cdot \zeta_{\sigma_{2}}(s)^{2}$.

Here $\sigma_{2}$ and $\sigma_{p}$ are elements of orders 2 and $p$, respectively, in the dihedral group $D_{2 p}$, where $p$ is an odd prime.

Since in these equations some zeta functions on the r.h.s. appear with the exponent 2, it follows that a zero of such zeta function is a multiple zero of $\zeta_{F}(s)$, provided it is not a zero of $\zeta_{E}(s)$.

To exclude the last possibility, we apply the well known asymptotic formula:

For a number field $M$ the number $N_{M}(T)$ of zeros (counting their multiplicities) of $\zeta_{M}(s)$ in the set $\{s \in \mathbb{C}: 0<\operatorname{Re} s<1,0<\operatorname{Im} s<T\}$ equals

$$
N_{M}(T)=\frac{(M: \mathbb{Q})}{2 \pi} \cdot T \log T+O(T) .
$$

In the case (i) $E$ is a proper subfield of $F^{\langle(12)\rangle}$, then, by (2), there are infinitely many zeros of $\zeta_{(12)}(s)$ in the critical strip, which are also zeros of $\zeta_{F}(s)$. Hence they have multiplicities at least 2 . In the cases (ii)-(iv) the argument is similar.

Remark. For the group $G=S_{4}$ the Brauer-Kuroda relation is

$$
\zeta_{F}(s) \cdot \zeta_{E}(s)^{2}=\zeta_{(12)}(s) \cdot \zeta_{(123)}(s) \cdot \zeta_{(1234)}(s),
$$

and we cannot apply the same argument as above.

On the other hand for this group we have

$$
\zeta_{F}(s)=\zeta_{K}(s) \cdot L\left(s, \chi_{1}, F / E\right)^{2} \cdot L\left(s, \chi_{2}, F / E\right)^{3} \cdot L\left(s, \chi_{3}, F / E\right)^{3},
$$

where $K=F^{A_{4}}$, and $\chi_{1}, \chi_{2}, \chi_{3}$ are irreducible characters of $G$ satisfying $\chi_{1}(1)=2$, $\chi_{2}(1)=\chi_{3}(1)=3$. Consequently, every zero of $\zeta_{F}(s)$, which is not a zero of $\zeta_{K}(s)$ is a multiple zero of $\zeta_{F}(s)$. There are infinitely many such zeros, by (2). 
Acknowledgement. I would like to thank Jerzy Kaczorowski for many enlightening discussions on the subject. I am indebted to Władysław Narkiewicz for informing me on papers $[\mathrm{To}]$ and $[\mathrm{W}]$.

\section{References}

[A] J.V. Armitage, Zeta functions with a zero at $s=\frac{1}{2}$, Inventiones Math. 15 (1972), 199-207.

[BBX] J. Browkin, J. Brzeziński, KeJian Xu, On exceptions in Brauer-Kuroda relations, Bull. Polish Acad. Sci. Math. 59 (2011), 207-214.

[F] A. Fröhlich, Artin-root numbers and normal integral bases for quaternion fields, Inventiones Math. 17 (1972), 143-166.

[H] H. Heilbronn, Zeta-Functions and L-Functions, in: J.W.S. Cassels, A. Fröhlich (eds.) Algebraic Number Theory, Chapter VIII, Academic Press, 1967, 204-230.

[LO] J.C. Lagarias, A.M. Odlyzko On computing Artin L-functions in the critical strip, Math. Comp. 33(147) (1979), 1081-1095.

[T] J. Tate, Stark's basic conjecture, IAS/Park City Mathematics Series 18 (2009), 9-31.

[To] E. Tollis, Zeros of Dedekind zeta functions in the critical strip, Math. Comp. 66(219) (1997), 1295-1321.

[W] L. Weinstein, The zeros of Artin L-series of a cubic field on the critical line, J. Number Theory 11 (1979), 279-284.

Address: Jerzy Browkin: Institute of Mathematics, Polish Academy of Sciences, Sniadeckich 8, PL-00-956 Warszawa, Poland.

E-mail: browkin@impan.pl

Received: 14 January 2013; revised: 27 February 2013 